\title{
Evaluación de los aprendizajes en escuelas rurales multigrado
}

\author{
Pilar Abós Olivares*, Roser Boix Tomás** \\ * Departamento de Ciencias de la Educación. Facultad de Ciencias Sociales y Humanas. Universidad de Zaragoza \\ ** Departamento de Didáctica y Organización Educativa. Facultad de Educación. Universidad de Barcelona
}

\section{RESUMEN}

La multigraduación como característica básica de las escuelas rurales exige unas determinados planteamientos didácticos, entre los que se incluye la evaluación. En este ámbito presentamos los resultados de una de las fases de un proyecto internacional de investigación ${ }^{1}$ en el que se estudiaron varias dimensiones sobre la enseñanza en las escuelas rurales. En el trabajo se presentan resultados referidos a una de las dimensiones estudiada, la evaluación, y al contexto español ${ }^{2}$. La metodología empleada para esta fase de la investigación fue de carácter cualitativo, a través de entrevistas estructuradas realizadas a maestros de aulas multigraduadas seleccionados a partir de sus respuestas a un cuestionario previo, y los resultados expuestos se apoyan en el análisis de contenido de las mismas a través de un sistema de categorización de carácter mixto. Los resultados y las posteriores conclusiones apuntan a la presencia de una variedad de instrumentos de evaluación, así como a estrategias de coevaluación entre pares.

Palabras Clave: Didáctica multigrado, Escuela rural, Metodologías activas, Evaluación de los aprendizajes.

\section{Evaluation of the learnings in rural multigrade schools}

\section{ABSTRACT}

Multigraduation as a basic characteristic of rural schools requires certain didactic approaches, including evaluation. In this area we present the results of one of the phases of an international project of investigation in which were studied several dimensions about the education in rural schools. In the work presented there are exposed the results referred to one of the dimensions studied, the evaluation, and in the Spanish context. The methodology used for this phase of the investigation has a qualitative character, across structured interviews realized to teachers of multigrade classrooms, selected from his answers to a previous questionnaire, and the exposed results rest on the analysis of content of the same ones across a system of categorization of mixed character. The results and the later conclusions point at the presence of a several instruments of evaluation, as well as at strategies of evaluation between couples.

Keywords: Didactic multigrade, rural School, active Methodologies, Evaluation of the learnings.

1 Proyecto EDU2009-13460, Subprograma EDUC (Ministerio de Ciencia e Innovación - España). Países participantes: España, Francia, Portugal, Chile y Uruguay.

2 El estudio se ha realizado en las Comunidades Autónomas de Andalucía, Aragón y Cataluña

\section{Marco teórico}

La multigraduación como referente teórico

La consideración de lo que entendemos por escuela rural es básica para contextualizar el planteamiento de este trabajo $\mathrm{y}$, en este sentido, la idea más importante es la de que las condiciones de la multigraduación, que no siempre van unidas a contextos rurales (Little, 2007), exigen de unas determinadas propuestas de enseñanza que tengan en cuenta aquellas peculiaridades orga- nizativas y didácticas que inciden en los procesos de aprendizaje del alumnado. Por ello los docentes deben superar algunas de las propuestas didácticas construidas únicamente para la enseñanza graduada y plantear otras más acordes con una enseñanza simultánea a varios grados, y que serán aquéllas que aumentan las probabilidades de logro de un aprendizaje activo e integral por parte del alumnado.

La multigraduación debe ser entendida como la forma natural de encuentro y trabajo conjunto entre niños de edades distintas. En este sentido Ellis, Rogoff y Cromer (1981) o Stone 
y Christie (1996) indican que los niños prefieren actuar con otros de diferentes edades en lugar de con niños de la misma edad y además, la heterogeneidad aumenta el interés y la motivación lo que se proyecta en una mayor participación activa en el aula. Las aportaciones del constructivismo social de $\underline{\mathrm{Vy}}$ gotsky (1985) inciden además en la importancia didáctica de la interacción social y la mediación entre pares en situaciones de aprendizaje.

Estas circunstancias han de ser tomadas como un valor pedagógico por parte del maestro, puesto que facilitan el uso de estrategias didácticas y de evaluación más acordes con una atención adecuada a la diversidad existente en la clase; el alumnado de diferentes edades puede ayudarse entre sí, aprender conjuntamente, aprender a aprender y enseñarse mutuamente, confiando en la fiabilidad propia del desarrollo humano (Strauss y Ziv, 2012) en el marco de situaciones sociales semejantes a las que se dan fuera de la escuela (Abós, Boix y Bustos, 2014). La escuela rural además, por sus características contextuales y por el papel que juega en la dimensión territorial, va a ser siempre conceptualizada desde una perspectiva de multigraduación y heterogeneidad, a no ser que cambien de forma radical las políticas educativas en relación al número de alumnos por aula y en el concepto de educación en el que se sustentan las mismas.

Para profundizar en el conocimiento de la realidad del proceso de enseñanza-aprendizaje de la escuela multigrado se planteó un trabajo de carácter internacional con la intención de saber si el modelo presente en dichas aulas, considerado a priori con alto valor pedagógico, pudiera ser extrapolable a otros contextos de enseñanza graduada. De este modo se plantearon objetivos generales relacionados con las estrategias didácticas, la organización del espacio y del tiempo (Abós y Bustos, 2015), los recursos y materiales didácticos (Boix y Bustos, 2014), y la evaluación del aprendizaje. La parte del trabajo que aquí se presenta está basada en el análisis de la dimensión evaluación del aprendizaje a través del análisis de contenido de las entrevistas realizadas a catorce maestros tutores de aulas multigrado, seleccionados según los resultados obtenidos en el cuestionario previo que indicaban una mayor presencia de metodologías activas y participativas apoyadas en principios de aprendizaje significativo, funcional y contextualizado.

La evaluación de los aprendizajes en el marco de las metodologías activo-participativas utilizadas en aulas multigrado

En términos generales hablar de escuela rural es hablar de escuela multigraduada, es decir de un espacio educativo en el que el proceso de enseñanza-aprendizaje es compartido por alumnado de diferentes edades y grados, convirtiéndose de este modo en un espacio idóneo para planteamientos pedagógicos inclusivos (Domingo, 2014). Unido a ellos, y desde la perspectiva del uso de metodologías activas- participativas, el fin de la evaluación es poder identificar procesos y productos que faciliten y demuestren el aprendizaje y el desarrollo personal y social del niño. Superar el modelo curricular de un aprendizaje por cursos y/o por edad y centrado en los resultados, supone poder recurrir a otro modelo de evaluación que incluya una gran variedad de procedimientos, instrumentos y agentes de evaluación que se adapten a distintas capacidades, niveles, intereses y posibilidades de expresión de los alumnos, es decir, que se adapten al aula multigrado. En este sentido la evaluación es un proceso dinámico, abierto y contextualizado que sirve para obtener información, formular juicios de valor y tomar decisiones (Pimienta, 2008). Las formas de trabajo de las metodologías activas, permiten una evaluación no centrada en los productos, sino en los procedimientos y en la aplicación de los procesos intelectuales superiores.

Además, la investigación ha revelado que la práctica de una evaluación puntual y final, con unos instrumentos que sólo permitan la repetición el máximo posible que lo que dicen los libros de texto o la misma exposición del maestro, puede inducir a una aprendizaje pasivo (Biggs, 1990), poco atractivo e incluso aburrido; mientras que una evaluación que acompañe el proceso de aprendizaje, una evaluación que valore de forma global los aprendizajes y que por si misma sea factor correctivo para seguir avanzando en la adquisición de nuevos conocimientos (Gibbs, 1992) permite la significatividad de éstos y da sentido al proceso de evaluación en el marco de la enseñanza. Esta es una condición para que la evaluación deje de centrarse exclusivamente en el producto y pase a considerar el proceso por el cual se llega a las respuestas, con incidencia en el grado con que los alumnos evidencian un aprendizaje (Cowley, 1989, cit. Ramsden, 1992).

La unión de multigraduación y estrategias didácticas activas se convierte en el tándem perfecto para la presencia de planteamientos evaluadores claramente alternativos a los tradicionales (exámenes escritos y orales), apoyados en planteamientos pedagógicos de búsqueda de aprendizajes significativos y construidos sobre el procesamiento individual y colectivo de la información. Planteamientos con criterios y procedimientos de evaluación basados en el proceso evaluación-aprendizaje-evaluación, en los que la misma evaluación es entendida como una valoración del aprendizaje y la relación asimétrica entre alumnos de distintas edades que aprenden dentro del aula contribuye al desarrollo de modalidades y tipos evaluación en los que participan diferentes agentes y que permiten la sistematización de los aprendizajes por delante de la fiscalización de los mismos. Evaluación en la que el intercambio entre iguales permite dar valor a las preguntas y no solo a las respuestas.

\section{Marco empírico}

\section{Objetivos y metodología de investigación}

En el marco general del trabajo (Domingo, Boix y Champo1lion, 2012; Domingo y Boix, 2015), del cual solo se presentan los resultados de una parte, se especificaron objetivos relacionados con el estudio de las prácticas docentes en las que estuviera presente el uso de metodologías activo-participativas, analizando cinco categorías: estrategias didácticas, organización del espacio y del tiempo, recursos didácticos y evaluación del aprendizaje del alumnado. En relación con el contenido del trabajo que presentamos el objetivo planteado fue peritar qué tipo de evaluación, en qué momento se utiliza, y con qué procedimientos e instrumentos, encontramos en las aulas multigrado, diferenciando así tres subcategorías momentos/tipos, agentes e instrumentos con sus correspondientes indicadores, y entendiendo que debía existir una relación entre el uso de estrategias didácticas activas y participativas y planteamientos evaluadores acordes con las mismas y, por lo tanto, diferentes a los considerados tradicionales.

De este modo se llevaron a cabo entrevistas semiestructuradas con preguntas referidas a la evaluación de los aprendizajes y que estaban en relación directa con las respuestas que los maestros entrevistados habían dado en los cuestionarios utilizados en la primera fase de la investigación en cuanto a la finalidad, los momentos y los instrumentos de la evaluación. En este sentido el contenido de las entrevistas se realizó de forma personalizada para cada uno de los docentes.

Algunos ejemplos de las preguntas formuladas son los siguientes: 
- Como indicas en el cuestionario, sueles utilizar diferentes técnicas de evaluación en clase no sólo exámenes. ¿Puedes explicar cómo lo aplicas?

- ¿De qué forma interviene el alumno en la evaluación? ¿Es sólo una cuestión del profesorado o el alumnado tiene un papel activo?

- ¿En qué momentos de la evaluación se utilizan los diferentes instrumentos y con qué objetivos?

- $(\ldots)$

La tabla 1 muestra los datos de las características de las aulas y los centros a los que pertenecían los docentes entrevistados, teniendo en cuenta que el dato fundamental de selección fue la multigraduación. El tratamiento de la información y el análisis del contenido de las entrevistas se realizaron a través del programa NVivo en el que se introdujo la información a partir de las cinco categorías iniciales a través de un sistema mixto inductivo que las dividía en subcategorías e indicadores.

\section{Resultados}

\section{Análisis de las entrevistas}

Dado que el equipo de investigación estaba constituido por investigadores de diferentes países, en el diseño y posterior desarrollo de la investigación se elaboró un glosario de términos que facilitasen la interpretación consensuada por parte de todos los miembros del equipo, tanto para el estudio cualitativo (entrevista) como para el etnográfico (observación participante). De esta manera se establecieron categorías (como ya hemos mencionado anteriormente) y dentro de éstas subcategorías e indicadores. En el caso de la categoría de evaluación, las subcategorías formuladas fueron: momentos, tipos, agentes de evaluación e instrumentos de evaluación.

En cada una de ellas se establecieron determinados indicadores vinculados a la utilización de metodologías activas y par- ticipativas. Así, en la subcategoría de momentos el indicador fundamental era el de la evaluación procesual como aquella que se realiza a lo largo del proceso de enseñanza-aprendizaje con carácter formativo y continuo y que permite tomar decisiones de mejora a lo largo del proceso. No obstante constatamos la presencia de indicadores clásicos como evaluación inicial, en muchas ocasiones con el mismo carácter que la final pero realizada al principio de una secuencia didáctica, y por supuesto final o sumativa destinada a la valoración de resultados y/o productos (López Pastor, 2006).

La subcategoría de tipos, vendría determinada por dos indicadores: evaluación criterial (entendida como una evaluación nomotética realizada en base a criterios previamente establecidos) y evaluación estándar (definida como evaluación externa y/o de diagnóstico que se realiza en determinados periodos de la escolarización de un alumno).

Por su parte, la subcategoría de agentes de evaluación estaba representada por tres indicadores: coevaluación (evaluación conjunta de una actividad realizada entre varios sujetos), autoevaluación (cuando un alumno evalúa su propio trabajo, de manera que el rol de evaluador y evaluado coinciden en las mismas personas) y heteroevaluación (cuando la evaluación la realiza una persona sobre otra, de forma que los evaluadores son distintos a las personas evaluadas).

En la subcategoría de instrumentos de evaluación, los indicadores que permitían interpretarla como vinculada a estrategias activas eran: escalas y pautas de observación, portafolio, registro anecdótico e informe. Instrumentos a los que añadir el examen escrito/oral y el cuaderno del alumno.

De acuerdo a todo ello los resultados se exponen en torno a las subcategorías formuladas: momentos y tipos ¿cuándo se evalúa?; agentes de evaluación ¿quién evalúa? e instrumentos de evaluación ¿cómo se evalúa?, indicando la presencia o no de aquellos indicadores referidos a las metodologías activas y participativas.

Tabla 1. Características de las aulas y centros

\begin{tabular}{|c|c|c|c|c|}
\hline \multirow{2}{*}{ Entrevista } & \multirow{2}{*}{ Cursos } & \multicolumn{2}{|c|}{ Tipo de centro } & \multirow[b]{2}{*}{ Comunidad } \\
\hline & & Agrupado $^{1}$ & No agrupado & \\
\hline 1 & $1^{\mathrm{o}}$ y $3^{\mathrm{o}}$ infantil; $2^{\mathrm{o}}, 3^{\mathrm{o}}, 4^{\mathrm{o}}$ y $6^{\mathrm{o}}$ primaria & $x$ & & Andalucía \\
\hline 2 & $1^{\mathrm{o}}, 2^{\mathrm{o}}$ y $3^{\mathrm{o}}$ primaria & $x$ & & Andalucía \\
\hline 3 & $3^{\circ}, 4^{\circ}, 5^{\circ}$ y $6^{\circ}$ primaria & $x$ & & Andalucía \\
\hline 4 & $1^{\mathrm{o}}$ y $2^{\mathrm{o}}$ primaria & $x$ & & Andalucía \\
\hline 5 & $3^{\circ}$ y $4^{\circ}$ primaria & & $x$ & Andalucía \\
\hline 6 & Unitaria & $x$ & & Aragón \\
\hline 7 & $3^{\circ}, 4^{\circ}$ y $6^{\circ}$ primaria & $x$ & & Aragón \\
\hline 8 & $1^{\mathrm{o}}, 2^{\mathrm{o}}$ y $3^{\mathrm{o}}$ infantil & & $x$ & Aragón \\
\hline 9 & $5^{\circ}$ y $6^{\circ}$ primaria & $x$ & & Aragón \\
\hline 10 & $1^{\mathrm{o}}, 2^{\mathrm{o}}, 3^{\mathrm{o}}$ y $4^{\mathrm{o}}$ primaria & $x$ & & Cataluña \\
\hline 11 & $3^{\circ}, 4,5, y 6^{\circ}$ primaria & $x$ & & Cataluña \\
\hline 12 & $3^{\circ}, 4^{\circ}, 5^{\circ}$ y $6^{\circ}$ primaria & $\mathrm{x}$ & & Cataluña \\
\hline 13 & $1^{\circ}, 2^{\circ}, 3^{\circ}$ y $4^{\circ}$ primaria & $x$ & & Cataluña \\
\hline 14 & Unitaria & $x$ & & Cataluña \\
\hline
\end{tabular}

\footnotetext{
${ }^{1}$ CPR en Andalucía, CRA en Aragón y ZER en Cataluña
} 
¿Cuándo se evalúa en las escuelas rurales multigrado?

La presencia de los tipos/momentos clásicos en la evaluación (inicial, continua y final) es un hecho en la escuela multigrado, aunque algunas de las reflexiones del profesorado entrevistado sobre los momentos/tipos de evaluación deben ser contextualizadas en el marco de la tipología de escuela a la que nos referimos, es decir, una escuela de pequeño tamaño, con un número reducido de alumnos por aula (tanto si el centro es unitario como cíclico) y con un contacto casi permanente con las familias que condiciona favorablemente la información para llevar a cabo una evaluación del proceso de los aprendizajes. El maestro está al día de los aprendizajes realizados por sus alumnos y, puede actuar en consecuencia, según las necesidades curriculares de los mismos.

"Hombre, pues la verdad es que por suerte al tener seis alumnos, la verdad es que se está evaluando todo el día... ya los ves todos los días quién va bien, quién va mal..." (Entrevista 6)

En esta evaluación del proceso, la evaluación inicial, el saber qué saben los alumnos del contenido a tratar, es un momento de sistematización que comparten la mayoría de los maestros entrevistados. Un momento del proceso que se refuerza con la obtención de información de cada uno de los alumnos, del curso anterior, de las pruebas especiales y/o de diagnóstico que hayan podido realizarse; sea como sea, la evaluación inicial, es el punto de partida de los maestros rurales y forma parte del proceso natural de aprendizaje de los alumnos.

\footnotetext{
"Siempre la evaluación inicial me sirve para saber de dónde parto, para saber en qué nivel están mis alumnos" (Entrevista 6)

Como evaluación inicial, en principio se organizan...bueno, se hacen tres cosas ¿no? Por un lado es la información que ya tienes de los cursos anteriores, que si ya han sido tus alumnos los conoces y si no te la pasa el compañero. Luego, aparte, se pasa lo que son las pruebas que trae el método, y también en ciertos casos se realizan pruebas especiales. (...) O que se considera que en esa prueba de la editorial no están acertadas... ampliarlas un poquito más. Pero todo esto se habla y se acuerda ¿no? a nivel de centro. Luego lo que es la evaluación continua...la que hacemos, siempre yo hago diferentes tipos de prueba ¿no? Hombre, está... Como técnica ya sabemos que la observación sistemática y diaria pero con la evaluación continua se utilizan también lo que son las pruebas que hago orales y escritas, y después el seguimiento de los cuadernos y del trabajo diario, que además en una unitaria es fundamental ¿no? Pero bueno, también les viene bien a los niños saber que hay unos momentos para controles especiales, que sepan lo que es pasar por eso ¿no? y el que sea un grupo pequeñito, lo que son las pruebas orales también porque me gusta irlos preparando para que se acostumbren a ir estudiando ¿no?, a ir organizando la información $y$ que poquito a poco vayan ellos...(...) Y la evaluación final, pues normalmente son el resultado de nota que tenemos un criterio de calificación también establecido desde el centro y yo llevo lo que es el diario de clase, que me sirve también de registro anecdótico (Entrevista 1).

“... nos hemos tomado muy en serio lo de la evaluación continua... por ejemplo al final de cada tema hay actividades que salen competencias, evaluación continua" (Entrevista 8)
}

La evaluación continua, es otro de los momentos fundamental en el proceso de evaluación llevado a cabo por los maestros entrevistados. Es importante destacar que es entendida como una evaluación formativa, en relación al progreso que cada uno de los alumnos hacen en su aprendizaje de modo que se identifican rápidamente los problemas más comunes o incluso se avanza, por parte del propio maestro, en cuales serán los problemas que le van a surgir a cada uno de ellos y en qué fase del proceso de aprendizaje. Sin embargo, aunque es entendida como evaluación formativa no siempre se aplica como tal y puede utilizarse simplemente como una forma de seguimiento diario, que intenta evitar el fracaso y la supuesta pérdida de tiempo que requiere volver a trabajar el contenido complejo, es decir, el poder seguir trabajando "con normalidad" sin interrupciones que puedan romper "el buen ritmo de aprendizaje" que subyace en la clase.

\section{¿Quién evalúa en las escuelas rurales multigrado?}

Teniendo en cuenta quién evalúa, se han encontrado experiencias de autoevaluación que son consideradas de un alto valor pedagógico por los docentes que las ponen en práctica.

\begin{abstract}
"Hay cosas que por ejemplo, eso que te he dicho de la tabla ellos mismos se evalúan y luego se comentan 'pues tengo que estudiármela más o..." (Entrevista 2).

"Hay actividades que las evaluamos sobre la marcha, ellos mismos para que ellos...por ejemplo la tabla. Pues luego vemos, 'a ver, ¿tu cuantos fallos has tenido?' 'Yo, ninguno'. 'Ah, muy bien'. Y, 'y yo cuatro, entonces, tengo que estudiar más'. Pues ya sabes ¿no? Luego al final del trimestre les doy yo una hojita donde ellos dan su opinión a un nivel, qué es lo que he aprendido, qué es lo que me ha costado menos trabajo, qué me ha costado menos, en qué tengo que mejorar... Entonces se autoevalúan ellos lo que es el trimestre en general. Luego eso se lo doy yo a los padres y muchas veces coinciden en todo conmigo" (Entrevista 3).
\end{abstract}

En otras ocasiones, se localizaron también experiencias de heteroevaluación y coevaluación que actúan de factor corrector del propio proceso de aprendizaje del alumno.

"La tabla se la pregunto por escrito y luego se la corrige...uno se la corrige al otro. Lo corregimos oralmente, pero yo... Es una hoja que tiene doce casillas. Entonces, yo les hago la pregunta y ellos ponen la respuesta solamente. Yo les marco el ritmo ¿no? Luego, cuando acabamos se lo vuelvo a repetir para que lo repasen y cuando acabamos yo les digo 'cambio' se cambian la hoja con el compañero, volvemos a repetir..." (Entrevista 3 ).

"Normalmente se hace, a ver, normalmente lo he hecho, por ejemplo, cuando hicimos el proyecto. Cuando lo terminamos hicimos una coevaluación ¿no? Bueno, pues a ver pues mira, él ha participado más, yo he participado más o menos, él ha realizado esto incluso con mi propio trabajo, a lo mejor pues mira, hemos hecho esto" (Entrevista 5).

"me gusta que se corrijan entre ellos... para fomentar la autoevaluación y luego la coevaluación también un poquito... les mando corregirse entre ellos" (Entrevista 6)

De todas formas, tanto la coevaluación como la heteroevaluación realizada por el alumnado, se plantean como situaciones de evaluación puntual, o incluso anecdótico. Si bien reconocen la facilidad con la que se pueden dar este tipo de evaluación debido al reducido número de alumnos, e incluso su valor pedagógico, no es una práctica habitual en los propósitos de evaluación de los maestros entrevistados, como un elemento más de su planificación didáctica.

¿Qué instrumentos se utilizan para evaluar en las escuelas rurales multigrado?

Los métodos tradicionales de recogida de información para la evaluación, fundamentalmente pruebas de carácter escrito, si- 
guen prevaleciendo como pruebas hegemónicas en la evaluación y en los esquemas previos de las familias para su potenciación, teniendo en cuenta que la calificación numérica sigue siendo obligatoria.

"El examen de toda la vida de un ocho, un nueve tal, tal. Pues hacemos todos los días, tienes el que trabaja en casa, tienes el que se muestra dispuesto en clase o el que ayuda al otro o una evaluación más valorativa, más de colaboración con los demás. Ya no sólo... que al final, lo que nos van a pedir es esto porque cuando vienen las familias: 'Bueno, ¿pero mi niño no ha sacado un diez?' En el examen un niño tenía un diez. 'Sí, señora. Pero es que, al margen de esto, pues hay otras cosas en la escuela... tal'. Al final, a la familia lo que agrada es el diez y lo que le duele es el cuatro" (Entrevista 2).

"tenemos un examen o test por cada tema" (Entrevista 6)

Y aunque para algunas de estas pruebas o exámenes, se recurre a materiales para evaluación elaborados por las propias editoriales, los docentes añaden pruebas de carácter oral, o adaptaciones relacionadas con las características del grupo.

"Entonces ellos se ven así quien ha estudiado más, quien ha estudiado menos y como lo hacemos oral, lo repasamos entre todos. Eso lo hacemos antes del examen, que les ponemos una pruebecilla. (...) La de la editorial" (Entrevista 3).

“...el tema de los exámenes claro, no cogerlos de las editoriales, te los tienes que hacer tú. Ahora lleva mucho trabajo pero el año que viene, en teoría no lo tendré que hacer" (Entrevista 6)

"Però sí que fem proves escrites $i$, fins $i$ tot, orals eh? I orals, sobretot... (Traducción: ("Pero sí que hacemos pruebas escritas e, incluso, orales ¿eh? Y orales, sobretodo....") (Entrevista 11)

Por otro lado, los docentes que trabajan en contextos rurales alternan estos instrumentos más tradicionales con otros recursos o herramientas de recogida de información, como los cuadernos de clase, los registros, y otros destinados a fomentar la lectura y la autogestión, como el pasaporte de lectura, donde quedan registrados los libros que cada alumno o alumna ha leído. Su presencia está claramente relacionada con el uso de la autoevaluación y la coevaluación y tienen un gran peso en la evaluación de contenidos comportamentales. En otras ocasiones es el diario de clase el que se utiliza como instrumento de seguimiento del alumnado permitiendo una recogida de información más rigurosa y sistematizada.

"Aquí tienes que tenerlo todo muy pensado y bien anotado en tu diario de clase para poder sacar lo mejor que puedas. Que luego no digan: 'Ay, ha llegado niño de..., hay que ver qué mal va el niño" (Entrevista 3).

"yo he marcado cuadernos de clase, exámenes escritos y registros anecdóticos... en los exámenes escritos manifestando el trabajo, lo que ha asimilado..." (Entrevista 9)

"los registros son cosas que a mí me han llamado la atención y las anoto en mi cuaderno" (Entrevista 9)

Además, el reducido número de alumnos, facilita al maestro que los registros de información sean rápidos y periódicos.

"Y como son tan pocos, de muchas cosas me acuerdo. Que no me hace falta ni apuntarlas. Me acuerdo o no me acuerdo, pero vamos que para evaluar a cuatro nada más..." (Entrevista 2).

"con los trabajos que han hecho los niños" (Entrevista 7)

"Y luego tú con la observación que vas haciendo en el aula lo vas evaluando" (Entrevista 7)
En ocasiones, la selección y variedad de los instrumentos está en estrecha relación tanto con los objetivos de la programación como con el momento en el que se lleva a cabo y, por lo tanto, acorde con la planificación diseñada.

"Si necessito un instrument el tinc, sigui per l'avaluació inicial o sigui per la contínua o això...La final, la final moltes...O sigui, inicial, a vegades és senzillament només a veure, a través d'unes preguntes que tu fas sobre els coneixements previs que puguin tenir $i$ ja està, $i$ que se'ls fa per escrit $i$ tu t'ho mires i treus les conclusions que això és així. La contínua sí que moltes vegades és, tinc una graella amb tota una sèrie d'items en els quals jo vaig anotant sobre allò que he volgut d'aquella unitat que vagin sabent o aixi, o amb l'observació de la llibreta o l'observació directa que tu tens a classe de les preguntes...de quan fas preguntes, de quan estem allò...de les aportacions que fan ells a les activitats o el que sigui, en vas prenent...La final, és el clàssic, o fas una prova o és a través d'un mapa conceptual..."(Traducción: "Si necesito un instrumento lo tengo, o sea para la evaluación inicial o sea para la continua o eso ... Al final, al final muchas ... O sea, inicial, a veces es sencillamente sólo a ver, a través de unas preguntas que tu haces sobre los conocimientos previsto que puedan tener y ya está, y que se les hace hacer por escrito y tu te lo miras y sacas las conclusiones que esto es así. La continua si que a veces es, tengo una parrilla con toda una serie de items en la que yo voy anotando sobre aquello que he requerido de aquella unidad que vayan sabiendo o así o con la observación de la libreta o la observación directa que tu tienes en la clase de las preguntas... de cuando haces preguntas, de cuando estamos en aquello... de las aportaciones que hace ellos a las actividades o lo que sea ... vas tomando ... Al final, es lo clásico, o haces una prueba o es a través de un mapa conceptual ..." (Entrevista 11)

\section{Análisis de las intersecciones}

Una de las ventajas de la utilización del programa NVivo para el análisis de contenido es la posibilidad que ofrece de establecer conexiones entre las subcategorías establecidas, tanto entre las pertenecientes a una misma categoría como a las otras. En este sentido cabe señalar, que el mayor número de intersecciones encontradas está presente con subcategorías de la categoría “Estrategias Didácticas".

Al analizar la categoría "Evaluación" destacamos los hallazgos en las intersecciones entre momentos (cuándo) con agentes (quién), momentos(cuándo) con instrumentos (cómo) y agentes (quién) con instrumentos (cómo). Sin embargo no se han encontrado entre las tres subcategorías de manera conjunta.

\section{Momentos + Agentes de Evaluación}

"Entonces, cuando acaba el día yo no les pongo la tarjeta, se la ponen ellos, bueno a veces se la tengo que poner yo porque se quieren poner la que no les corresponde ino? pero entonces, dicen "amarillo es la mala y la verde es la buena" "pues yo amarilla porque he trabajado poco" o porque he hablado mucho o porque... Entonces ellos se evalúan, es que son muy revoltosos y yo ya no sabía qué hacer con ellos, nos inventamos ese mecanismo y parece que funciona"

"Perquè fem molts tipus d'avaluacions i els criteris, que els teníem més escrits des de feia molts anys, com a pura avaluació, $i$ nosaltres el que fem més ara, veiem que és més...és avaluació-aprenentatge, aprenentatge-avaluació, avaluació-aprenentatge. No és... mai no hi ha, si ens n'hem adonat que tot, fins i tot la pròpia avaluació, és aprenentatge, valoració, segueixo, milloro no, doncs torno. Afegeixo cosa nova, què tal? La sé, ho sé, no sé, torno..."(Traducción: "Porque hacemos muchos tipos de evaluaciones y los criterios, 
que los teníamos por escrito desde hace muchos años, como pura evaluación, y nosotros lo que hacemos ahora, vemos que es más... es una evaluación -aprendizaje, aprendizaje-evaluación, evaluación-aprendizaje. No es... nunca hay, si no hemos dado cuenta que todo, incluso la propia evaluación es aprendizaje, valoración, sigo, mejoro no, pues vuelvo. Añado lo que es nuevo, ¿qué tal ?La sé, lo sé, vuelvo...")

"Por ejemplo, había una niña que al principio no hablaba casi por desconocimiento del idioma y ya ves que se relaciona con todos y que sabe pedirte las cosas pues, día a día. Esa evaluación tú ves que va evolucionando su lenguaje. Más que nada es así. Por eso digo que con la observación porque aquí en infantil claro, exámenes no pero sí que en el trabajo que van realizando día a día tú vas mirando el progreso que vas viendo..."

Las intersecciones encontradas entre Momentos y Agentes de Evaluación refuerzan el planteamiento de que en la evaluación el papel del alumno como agente toma un carácter significativo, tanto si el momento es al final del proceso como inicial y/o continuo. Además, el hecho de llevar a cabo diferentes tipos de evaluaciones y formular criterios diversos supone para el maestro un estar en constante búsqueda de nuevas formas que le permitan adaptarse a la realidad pedagógica del aula multigrado. A pesar que en ésta, el número de alumnos es reducido, el maestro comprometido intenta ir más allá del momento y el agente, y construye un proceso de reflexión (valoración, mejora, revisión, etc.) compartiendo las inquietudes grupales y/o individuales con sus alumnos sin obviar sus motivaciones.

\section{Momentos + Instrumentos de Evaluación}

"Entonces, siempre hay que pasar una serie de pruebas, les pides trabajos, deberes y tal pero bueno, ya los ves todos los días quién va bien, quién va mal, dónde hay apoyo cuando necesitan algo..."

"Y luego pues siempre tenemos los, a nivel de ciclo lo decidimos, tenemos un examen o test por cada tema y luego pues hombre, siempre lo que son los trabajos me los tienen que reenviar a mí yo los corrijo, tengo una nota para trabajos, para trabajo en clase, para trabajos exteriores y luego a nivel de actitud y de comportamiento todo eso llevo siempre un pequeño registro. Aunque ya te digo, la verdad es que hay muchas veces que no lo necesitas. Tienes allí un montón de notas pero los vas conociendo y los de sexto ya son dos años y la verdad es que ya los conoces casi..."

"Mira pues, lo que son los instrumentos de evaluación y los tipo de evaluación utilizados, eso es un acuerdo de la escuela ¿no? O sea, es que eso está dentro del proyecto y lo hacemos así. Como evaluación inicial (...). Luego lo que es la evaluación continua (...). Y la evaluación final (...)".

"Siempre es flexible pero era para tener un poquito de juego también por no sólo evaluar por las notas de los exámenes porque todos sabemos que los niños en los exámenes pueden fallar y no sólo evaluar en base a un que si el niño como es muy bueno y se comporta muy bien le tenemos que poner..."

El examen de toda la vida que ---el examen de toda la vida de un ocho, un nueve tal, tal. Pues hacemos todos los días, tienes el que trabaja en casa, tienes el que se muestra dispuesto en clase el que ayuda al otro o una evaluación más valorativa, más de colaboración con los demás.

El examen traspasa el concepto de instrumento de evaluación en el aula multigrado. Es importante destacar cómo las familias obligan al maestro que trabaja en la escuela rural a cuantificar el aprendizaje de sus alumnos a pesar de no ser en absoluto necesario la realización de este tipo de pruebas, y mucho menos, cuando el número de alumnos es tan reducido que, como hemos visto anteriormente, el maestro conoce la situación de aprendizaje en la que se encuentra cada uno de sus alumnos.

"O sigui, una de les avantatges que té és que com que hi ha nens transparents, perquè de tots en saps alguna cosa, són suficients com perquè ho puguis saber, tu ja saps què és el que saben, què és el que no saben, què és el que han après, quines estratègies han fet servir, si són capaços de..."(Traducción: "O sea, una de las ventajas que tiene es que como hay niños transparentes, porque de todos sabes alguna cosa, son suficientes como para que lo puedas saber, tu ya sabes que es lo que saben, qué es lo que no saben, qué es lo que han aprendido, qué estrategias han utilizado, si son capaces de ...")

Las familias requieren de un examen para poder "puntuar" el aprendizaje de sus hijos, más allá de si han llevado a cabo otro tipo de actividades que conlleven un aprendizaje cualitativo; la comunidad educativa, y especialmente las políticas educativas deben reflexionar sobre este hecho si realmente quieren implantar metodologías alternativas a la tradicional; se pueden desarrollar estrategias didácticas y de evaluación distintas a las clásicas, pero no podemos obviar que las familias, el mosaico social en el que se sitúa la escuela, sigue exigiendo de un número para determinar el grado de aprendizaje de los alumnos.

Ya no sólo...que al final---lo que nos van a pedir es esto, porque cuando vienen las familias "bueno, ¿pero mi niño no ha sacado un diez?" en el examen un niño tenía un diez "Sí señora pero es que al margen de esto pues hay otras cosas en la escuela, tal" al final a la familia lo que agrada es el diez y lo que le duele es el cuatro".

\section{Agentes de Evaluación + Instrumentos}

"Y claro, si tú haces un examen que lo corrija, eso de poner mal... Corrígelo, corrígelo tú porque si luego ya lo corriges y ya después de otro apoyo y de otro refuerzo ya lo sabes hacer pues (...) si has cumplido que al final uno lo sabe pues eso evalúas..."

A las intersecciones anteriores podemos añadir algunas otras encontradas entre las subcategorías de evaluación y otras pertenecientes a otras categorías (momentos con planteamiento pedagógico y con actividades; instrumentos con planteamiento pedagógico y con método; y agentes con actividades), lo que supone una mayor coherencia entre los distintos elementos presentes en la práctica docente de los maestros entrevistados.

De este modo, la evaluación por pares, entre alumnos, la coevaluación de actividades que no están programadas para ser evaluadas permiten al alumno, de edades distintas y de la misma edad, reflexionar conjuntamente sobre la tarea realizada; en esta línea, hay dos aspectos importantes a considerar; el trabajo colaborativo entre los alumnos, fomenta la autonomía de aprendizaje, y aprenden a aprender; por otra parte, en el aula multigrado, el maestro también puede atender a sus alumnos de forma individualizada a pesar que siempre se ha dado una imagen del aula rural como aquella en la que el maestro no dispone de tiempo para sus alumnos por ser tan diversos; es simplemente una cuestión de organización y de entender que el docente no es el protagonista del proceso de enseñanza-aprendizaje.

\section{Conclusiones}

Las conclusiones que se exponen se fundamentan en los resultados que se acaban de presentar, fruto de procedimientos cualitativos de investigación y con una selección condicionada de docentes. Por ello en ningún caso se busca la generalización 
de resultados sino la profundización en el conocimiento de las prácticas pedagógicas utilizadas en aulas multigrado.

En cuanto al sentido global de la evaluación se entiende como un proceso que parte de lo que el alumnado sabe y que el menor número de niños por aula favorece una evaluación diaria, ésta no tiene siempre el carácter de evaluación continua ya que suele estar más centrada en los resultados que en el proceso. En este sentido, si bien se da una evaluación a lo largo de los procesos de enseñanza-aprendizaje que podría remitir a una evaluación entre iguales o a una evaluación que se realiza por observación directa y por una valoración diaria de las producciones de los alumnos, la evaluación que determina la calificación, es decir, la justificación y el rendimiento de cuentas respecto a la adquisición de los contenidos curriculares se centraliza en momentos muy concretos (finalización de las unidades didácticas/ temas y finalización de los trimestres). Aunque la lógica sea evaluación-aprendizaje, aprendizaje evaluación y el aprendizaje sea más valorado que evaluado, la justificación externa del producto sigue teniendo el mayor peso; una evaluación apoyada en las respuestas del alumnado, a pesar de la importancia que puede tener la evaluación basada en las preguntas ( $\underline{\text { Santos, 2014)}) . ~}$

La presencia de lo que podríamos interpretar como evaluación continua no se debe a criterios de elección sino más bien al hecho de tener pocos alumnos en el aula.

El docente sigue siendo el principal agente de la evaluación, y aunque el alumnado como agente evaluador está presente en algunas dinámicas en las que se hace referencia a la evaluación entre iguales, ésta queda limitada, en muchas ocasiones, a la corrección de ejercicios en la mayoría de los casos entre alumnado del mismo nivel. Los "conatos" de autoevaluación y de coevaluación están provocados muchas veces por las propias necesidades del docente de atender a otros alumnos del grupo, que por la creencia en sus posibilidades educativas, es decir se utilizan en las aulas rurales, pero no siempre con intencionalidad evaluadora para la sistematización y valoración del aprendizaje por parte del maestro.

Algunos instrumentos más creativos, como el sistema de tarjetas, los diarios de clase o los registros individuales y colectivos tanto de comportamiento como del trabajo diario, se presentan como alternativa a la hegemonía de los métodos tradicionales de evaluación. Además la diversidad de instrumentos se considera idónea para adecuar la evaluación a las circunstancias variables y diversas del aula multigrado. No obstante, se percibe cómo las familias de alguna manera "esperan" el examen y la calificación, y cómo los instrumentos utilizados (muchas veces facilitados por las propias editoriales) están enfocados a esa secuencia.

Los criterios de evaluación que se siguen son comunes para todas las unidades y semejantes a los de los centros graduados, aunque su nivel de adaptación no sea el adecuado, ya que están apoyados en los ofrecidos por las editoriales y van dirigidos exclusivamente a tener en cuenta diferentes aspectos curriculares además de las pruebas.

En cierto modo, los maestros se sienten comprometidos a la hora de garantizar el éxito de sus alumnos en momentos concretos y a través de un instrumento de evaluación específico cuyo principal sentido es la acreditación y la rendición de cuentas. En otras palabras, los maestros se sienten divididos entre una "evaluación auténtica", pero informal, que desarrolla diariamente, a lo largo del curso y sin implicaciones externas al aula, y una evaluación institucional y formal, bajo la que se establece una correlación directa entre su propio trabajo y el rendimiento de "sus" alumnos reflejado a través de la evaluación institucional. Así, mientras en la "evaluación auténtica" los maestros cuestionan e indagan estratégicamente sobre el desarrollo y los procesos de aprendizaje de los alumnos, en la evaluación institucional tiende a preocuparse por que la fotografía de sus alumnos no terminen por implicar el cuestionamiento externo de su labor docente.

En general se considera que la evaluación debe ser compartida, aunque no lo lleven a cabo, y que es muy compleja, lo que precisa de una mayor dedicación apoyada en una sólida formación.

Existe una brecha entre las evaluaciones externas e institucionales con las propias, ya que las primeras no terminan realmente de cumplir la función la función de una evaluación del proceso auténtica donde pueda servir para mejorar las debilidades propias del sistema. Por lo que en cierto modo el docente se siente en la obligación de garantizar resultados positivos en momentos puntuales del propio proceso de E-A y entre ellos los de la evaluación externa.

Por otro lado, se observa el peso que han tomado las evaluaciones institucionales estandarizadas en la escuela, cómo condicionan los ritmos de enseñanza y aprendizaje como un elemento cada vez más regulado interna y externamente.

Todo ello nos lleva a señalar la importancia de la estrecha relación de la evaluación con el resto de elementos didácticos presentes en el aula (contenidos, recursos, espacios...) y de la necesidad de una mayor investigación de su especificidad en aulas multigrado.

\section{Algunas limitaciones del estudio}

Las limitaciones que se han presentado en este estudio vienen estrechamente relacionadas con las limitaciones propias de una investigación de corte cualitativo en el que se presenta sólo una parte de la misma, y en este caso, como se ha expuesto, concretado a las entrevistas realizadas a 14 maestros rurales de tres comunidades autónomas españolas en el marco de una macroinvestigación internacional de carácter mixto. En este sentido, no pueden generalizarse, como avanzábamos en las conclusiones, los resultados obtenidos más allá del contexto en el que se sitúa el objeto de estudio, con lo que la representatividad de los datos queda sujeta a información dada por los maestros entrevistados y a su posterior carácter interpretativo contrastado con el conocimiento científico que existe en relación a la temática; y en relación a ello, ésta ha sido otra limitación importante en esta investigación: la falta de conocimiento científico sobre la propia temática. Son muy escasos los estudios científicos de referencia, tanto a nivel nacional como internacional, que permitan sustentar bajo un marco teórico válido y fiable, el tema de estudio. A pesar que existen muy buenas prácticas y experiencias innovadoras al respecto, éstas no siempre vienen avaladas por estudios rigurosos sobre la evaluación en la escuela rural bajo una perspectiva de metodología activa-participativa. Incluso los maestros entrevistados mostraban su preocupación por la falta de este conocimiento, que no les permite avanzar en su reflexión y posterior práctica sobre cómo, y que instrumentos de evaluación son más acordes con el proceso de enseñanza-aprendizaje-evaluación en la metodología activo-participativa.

\section{Referencias bibliográficas}

Abós, P y Bustos, A (2015). Teaching Strategies and Space Organisation in Multigrade Classrooms. Sisyphus, Journal of Education, 3(2), 58-77.

Abós, P, Boix, R. y Bustos, A (2014). Una aproximación al concepto pedagógico de aula multigrado. Aula de Innovación Educati$v a, 229,12-17 . B i g g s$, J.B. (1990). Teaching for desired learning outcomes. En N. Entwistle (Ed.) Handbook of educational ideas and practices. London y New York: Routledge. 
Boix, R y Bustos, A (2014). La enseñanza en las aulas multigrado: Una aproximación a las actividades escolares y los recursos didácticos desde la perspectiva del profesorado. Revista Iberoamericana de Evaluación Educativa, 7(3), 29-43.

Domingo, L. (2014). Contribucions pedagògiques de l'escola rural. La inclusió a les aules multigrau: un estudi de cas. Tesi Doctoral. Universitat de Vic.

Domingo, L., Boix, R. y Champollion, P. (2012). Estado de la cuestión del Proyecto de Investigación Educativa "La eficacia y la calidad en la adquisición de competencias que caracterizan a la escuela rural: $¿$ Es un modelo transferible a otra tipología de escuela". Educaçao, Santa María, 37(3), 425-436.

Domingo, L. y Boix, R. (2015). What can be learned from Spanish rural schools? Conclusions from an international project. International Journal of Educational Research. 74, 114-126.

Ellis, S., Rogoff, B. y Cromer, C. (1981). Age segregation in children's social interactions. Developmental Psychology, 17 (4), 399-407. Disponible en: http://dx.doi.org/10.1037/0012$\underline{1649.17 .4 .399}$
Gibbs, G. (1992). Improving the quality of student learning: theory and practice. Bristol: TES.

Little, A.W. (2007) Education for All and Multigrade Teaching: challenges and opportunities. Springer: London.

López Pastor, V. M. (Coord.). (2006). La evaluación en educación física. Revisión de los modelos tradicionales y planteamiento de una alternativa: La evaluación formativa y compartida. Buenos Aires: Miño y Dávila.

Pimienta, J. (2008). Evaluación de los aprendizajes. Un enfoque basado en competencias. México: Pearson.

Santos, L. (2014). Evaluación de aprendizajes en grupos multigrado de escuela rural. Aula de Innovación Educativa, 229, 2327.

Stone, S. J., y Christie, J. F. (1996) Collaborative literacy learning during sociodramatic play in a multiage (K-2) primary classroom. Journal of Research in Childhood Education, 10(2), 123-133.

Strauss, S. y Ziv, M. (2012): Teaching is a natural cognitive: ability for humans. Mind, Brain and Education, 6(4), 186-196.

Vygotsky, L.S. (1985). Pensée et langage. Paris: Editions Socials. 